\title{
Direct admission to the intensive care unit from the emergency department and mortality in critically ill hematology patients
}

Olivier Peyrony ${ }^{1 *}$ (10, Sylvie Chevret ${ }^{2,3,4}$, Anne-Pascale Meert ${ }^{5}$, Pierre Perez ${ }^{6}$, Achille Kouatchet ${ }^{7}$, Frédéric Pène ${ }^{4,8,9}$, Djamel Mokart ${ }^{10}$, Virginie Lemiale ${ }^{11}$, Alexandre Demoule ${ }^{12,13,14}$, Martine Nyunga ${ }^{15}$, Fabrice Bruneel ${ }^{16}$, Christine Lebert ${ }^{17}$, Dominique Benoit ${ }^{18}$, Adrien Mirouse ${ }^{11}$ and Elie Azoulay ${ }^{3,4,11}$

\begin{abstract}
Background: The aim of this study was to assess the benefit of direct ICU admission from the emergency department (ED) compared to admission from wards, in patients with hematological malignancies requiring critical care.

Methods: Post hoc analysis derived from a prospective, multicenter cohort study of 1011 critically ill adult patients with hematologic malignancies admitted to 17 ICU in Belgium and France from January 2010 to May 2011. The variable of interest was a direct ICU admission from the ED and the outcome was in-hospital mortality. The association between the variable of interest and the outcome was assessed by multivariable logistic regression after multiple imputation of missing data. Several sensitivity analyses were performed: complete case analysis, propensity score matching and multivariable Cox proportional-hazards analysis of 90-day survival.

Results: Direct ICU admission from the ED occurred in 266 (26.4\%) cases, 84 of whom (31.6\%) died in the hospital versus $311 / 742$ (41.9\%) in those who did not. After adjustment, direct ICU admission from the ED was associated with a decreased in-hospital mortality (adjusted OR: $0.63 ; 95 \% \mathrm{Cl} 0.45-0.88$ ). This was confirmed in the complete cases analysis (adjusted OR: 0.64; 95\% Cl 0.45-0.92) as well as in terms of hazard of death within the 90 days after admission (adjusted HR: $0.77 ; 95 \% \mathrm{Cl} 0.60-0.99$ ). By contrast, in the propensity score-matched sample of 402 patients, direct admission was not associated with in-hospital mortality (adjusted OR: 0.92; 95\% Cl 0.84-1.01).

Conclusions: In this study, patients with hematological malignancies admitted to the ICU were more likely to be alive at hospital discharge if they were directly admitted from the ED rather than from the wards. Assessment of early predictors of poor outcome in cancer patients admitted to the ED is crucial so as to allow early referral to the ICU and avoid delays in treatment initiation and mis-orientation.
\end{abstract}

Keywords: Emergency department, Direct admission, Intensive care unit, Hematological malignancy

\section{Background}

One of the goals of emergency physicians is to detect, based on clinical characteristics and physiological derangements, patients without established organ dysfunction, but who are at high risk of early life-threatening complications. Such patients should be early admitted to

\footnotetext{
${ }^{*}$ Correspondence: o.peyrony@hotmail.fr

1 Emergency Department, Hôpital Saint-Louis, 1 avenue Claude Vellefaux, 75010 Paris, France

Full list of author information is available at the end of the article
}

the intensive care unit (ICU) or high dependency units as to benefit from close monitoring. Indeed, when these patients are admitted to classic wards, the level of monitoring may be inappropriate. Moreover, the workload that these high-risk patients impose to clinicians also hampers optimal management of remaining patients. The occurrence of complications in wards could lead to delays in patient optimal care and transfer to the ICU and, therefore, increase morbi-mortality [1]. This is even more likely for immunocompromised patients in whom 
the risk of deterioration is higher and less easily predictable [2].

Thus, we hypothesized that immunocompromised patients admitted to the ICU directly from the emergency department (ED), were admitted sooner and, may have better outcomes compared to similar patients, admitted to ICU from the wards.

The aim of this study was to assess the benefit of direct ICU admission from the ED compared to admission from wards, in patients with hematological malignancies requiring critical care.

\section{Methods}

This is a post hoc analysis derived from a prospective, multicenter cohort study of 1011 critically ill adult patients with hematologic malignancies admitted to 17 ICUs in Belgium and France from January 1, 2010 to May 1, 2011 [3]. Briefly, the study was carried out in university or university-affiliated centers in France and Belgium that belonged to a research network instituted in 2005. In all 17 centers, a senior intensivist and a senior hematologist are available around the clock and make ICU-admission decisions together. During the study period, consecutive patients having hematologic malignancies who were admitted to the participating ICUs for any reason were included. Exclusion criteria were complete cure of the malignancy for more than 5 years, ICU admission only to maximize safety of a procedure, and age younger than 18 years. For this post hoc analysis, patients with missing data on the outcome were also excluded. The study was approved by the appropriate ethics committees in France and Belgium. All patients or relatives were informed and consented to participate in the study.

\section{Data collection and outcome measure}

Data abstracted from the study database were: age, sex, underlying malignancy, disease status (newly diagnosed if malignancy was diagnosed in the last month, complete or partial remission, other), autologous or allogeneic bone marrow or hematopoietic stem-cell transplantation (BMT/HSCT), treatment with long-course corticosteroids, Charlson comorbidity index, performance status, existence of organ failure based on the Sequential-related Organ Failure Assessment (SOFA) criteria, SOFA score, main reason for ICU admission, circumstances of ICU admission, time between hospital and ICU first request, and between first request and ICU admission, number of requests before ICU admission, specialty and experience of the physician that requested ICU admission, direct admission from the ED, length of ICU and hospital stay, vital status at ICU, at hospital discharge, and at day 90.

The variable of interest was direct admission from the ED, and the main outcome was in-hospital mortality.

\section{Statistical analysis}

Results are reported as medians with interquartile ranges (IQR) for continuous variables and numbers with percentages for binary and categorical variables.

Patient characteristics were compared using the Chisquare test for categorical variables and the Wilcoxon rank sum test or the Student $t$ test, as appropriate, for continuous variables (with normality tested with Shapiro-Wilk test). We investigated the association between the variable of interest and the outcome by multivariable logistic regression to search for potential confounders. Characteristics associated with the outcome on the basis of $P$-values less than 0.1 by univariable analyses were included in a multivariable logistic model; clinically relevant prognostic characteristics such as SOFA score, Charlson risk index or performance status, were forced in the model regardless of their $P$ value. Then, a backward selection procedure was applied, except for clinically relevant prognostic variables that were not removed from the model.

Missing data were managed with multiple imputation by chained equations [4]. The distribution of the data according to the presence or absence of missing data was checked (plots if continuous or tables if categorical variables) to ensure that missing data were missing completely at random. As recommended [5], variables included in the imputation model were those of the logistic regression prediction model (including the outcome), in addition to auxiliary covariates correlated with the missing variables (i.e., sex, underlying disease, days since diagnosis, days between first call to intensivist and ICU admission, experience of the physician requesting ICU, number of calls before ICU admission, organ failures). Five datasets were imputed with 50 iterations each. Multivariable logistic regression model was applied to the 5 imputed datasets and final estimates were obtained by averaging the 5 estimates according to Rubin's rules.

Several sensitivity analyses were performed. First, a complete cases analysis was performed. Second, to handle potential residual confounding by indication, a propensity score matching was done, where propensity score of being directly ICU admitted was estimated from a multivariable logistic model, with resulting balances in confounders checked by standardized mean differences and c-index [6], then matching performed without replacement within a caliper of 0.2 standard deviation of the logit of propensity score [7]. Third, we plotted survival Kaplan-Meier curve (from ICU admission to 90 days) according to direct admission status from the ED. Hazards ratio (HR) from Cox proportional-hazards models was used to quantify the association between the direct admission status and the outcome, adjusting for baseline predictors of survival. Underlying assumptions 
of the Cox model were checked: Proportional hazards $(\mathrm{PH})$ assumption was tested using a formal test based on the Schoenfeld residuals, with time-dependent effect considered for covariates that violated the $\mathrm{PH}$ assumption [8]. Log-linearity between non-binary covariates and hazard was assessed through splines; in case of nonlinear effect, covariates were dichotomized according to thresholds derived from the splines.

All $P$-values were two-sided, with values of 0.05 or less considered as statistically significant.

Data were analyzed with R 3.5.0 software (the R Foundation for Statistical Computing, Vienna, Austria).

\section{Results}

\section{General characteristics}

During the study period, 1011 patients were enrolled. Due to missing data on the outcome, 3 patients were excluded leading to a number of 1008 included patients in the analysis. Table 1 shows the patients' characteristics at ICU admission.

Patients were mostly men $(60.7 \%)$ and half were aged of less than 60 years. The most frequent malignancy was non-Hodgkin's lymphoma (31.6\%), followed by acute myeloid leukemia (27.2\%) and myeloma (12.5\%). Chronic malignancy as chronic lymphocytic or myeloid leukemia or myelodysplastic syndrome was present in 13.9\% of the patients. Disease status was complete or partial remission or newly diagnosed for $64.3 \%$ of the patients. One hundred and ninety-eight patients (19.8\%) had poor performance status. The most frequent main reasons for ICU admission were acute respiratory failure (39.2\%) and sepsis (26.8\%). Patients had respiratory failure in $62.7 \%$ cases, cardiovascular failure in $42.3 \%$ cases, renal failure in $30.3 \%$ cases, and neurological failure in $22.4 \%$ cases.

\section{Direct ICU admission from the ED and hospital mortality}

Table 2 shows the modalities of ICU admission. ICU admission was requested by the hematologist in 59.3\% of the cases and by an emergency physician in $22 \%$ of the cases. Physicians requested ICU transfer in the 4 [0-17] days after patient hospital admission. Patients were admitted to ICU most of the time by the day of the request (97.6\%) and after one call to the intensivist (87.7\%). Direct ICU admission from the ED occurred for 266 (26.4\%) patients (Fig. 1). Patients stayed in ICU 5 [2-11] days in median. ICU and in-hospital mortality were $27.7 \%$ and $39.2 \%$, respectively.

By univariable analyses (Additional file 1), variables associated with in-hospital mortality were an age $>60$ ( $44 \%$ vs. $34.6 \%$; $P=0.003$ ), non-complete or non-partial remission or non-newly diagnosed disease status (45.6\% vs. $36.2 \%$; $P=0.005$ ), allogeneic $\mathrm{BMT} / \mathrm{HSCT}$ recipient
(52.1\% vs. $36.9 \% ; P<0.0001)$, a high Charlson comorbidity index (median: 4; IQR: 3 to 6 vs median: 4; IQR: 2 to $5 ; P=0.0001)$, a poor performance status $(57.1 \%$ vs. $34.7 \%$; $P<0.0001$ ), a high SOFA score (median: 7; IQR 5 to 11 vs median: 5; IQR 3 to $7 ; P<0.0001$ ), time between hospitalization and first call to the intensivist (median: 7; IQR 1 to 21 vs median: 2; IQR 0 to $14 ; P<0.0001$ ), an ICU admission requested by a non-emergency physician $(41.3 \%$ vs. $31.5 \%$; $P=0.01)$. Conversely, direct ICU admission from the ED was associated with decreased in-hospital mortality $(31.6 \%$ vs. $41.9 \% ; P=0.004)$. Table 3 reports the estimated effect of direct ICU admission on the in-hospital mortality adjusted on prognostic variables, based on the multivariable logistic model after multiple imputation. Direct ICU admission from the ED was associated with decreased in-hospital mortality (OR: 0.63 ; $95 \%$ CI 0.45 to 0.88 ).

\section{Sensitivity analyses}

Figure 2 summarizes the estimated effects of direct ICU admission from the ED on in-hospital mortality depending on the sensitivity analysis.

First, the complete cases analysis confirmed the improved survival of direct ICU admission (OR: 0.64; 95\% CI 0.45-0.92).

Secondly, based on propensity score, 201 patients admitted to the ICU directly from the ED were matched with patients admitted from wards. Additional file 2 reports differences in patient characteristics according to location prior to ICU admission before matching and Additional file 3 shows the absolute mean differences in patient characteristics before and after matching on propensity score. In the matched sample of 402 patients, direct admission was not associated with in-hospital mortality (OR: 0.92; 95\% CI 0.84-1.01).

Last, survival was significantly improved in patients who were admitted directly to the ICU from the ED (Fig. 3), with an estimated unadjusted HR at 0.75 (95\% CI $0.59-0.94, P=0.014)$. After adjustment on survival predictors (namely, disease status, BMT/HSCT, Charlson comorbidity index, performance status and SOFA score at ICU admission), the adjusted HR was 0.77 (95\% CI 0.60 to $0.99, P=0.04$ ).

\section{Discussion}

In this post hoc analysis derived from a prospective, multicenter cohort study of 1011 critically ill adults patients with hematologic malignancies admitted to ICU, direct admission from the ED was associated with decreased in-hospital mortality in comparison with admission from the wards. This effect on mortality was confirmed in most of the sensitivity analyses except after matching on propensity score. 
Table 1 Patients' characteristics at ICU admission

\begin{tabular}{|c|c|c|c|c|}
\hline \multirow[t]{2}{*}{ Characteristics } & \multirow{2}{*}{$\begin{array}{l}\text { Overall cohort } \\
(N=1008)\end{array}$} & \multicolumn{2}{|c|}{ Status at hospital discharge } & \multirow[t]{2}{*}{ Missing data } \\
\hline & & Alive $(n=613)$ & Death $(n=395)$ & \\
\hline Age, median [IQR], years & 60 [49-69] & $59[47-68]$ & $62[52-71]$ & 0 \\
\hline Female gender, $n(\%)$ & $396(39.3)$ & $252(41.1)$ & $144(36.4)$ & 0 \\
\hline Underlying malignancy, $n$ (\%) & & & & 0 \\
\hline Non-Hodgkin's lymphoma & $319(31.6)$ & $190(31.0)$ & $129(32.7)$ & \\
\hline Acute myeloid leukemia & $274(27.2)$ & $162(26.4)$ & $112(28.4)$ & \\
\hline Myeloma & $126(12.5)$ & $86(14.0)$ & $40(10.1)$ & \\
\hline Chronic lymphocytic leukemia & $75(7.4)$ & $45(7.3)$ & $30(7.6)$ & \\
\hline Acute lymphocytic leukemia & $73(7.2)$ & $44(7.2)$ & $29(7.3)$ & \\
\hline Myelodysplastic syndrome & $46(4.6)$ & $26(4.2)$ & $20(5.1)$ & \\
\hline Hodgkin's disease & $25(2.5)$ & $18(2.9)$ & $7(1.8)$ & \\
\hline Chronic myeloid leukemia & $19(1.9)$ & $14(2.3)$ & $5(1.3)$ & \\
\hline Other & $51(5.1)$ & $28(4.5)$ & $23(5.8)$ & \\
\hline Disease status, $n(\%)$ & & & & 54 \\
\hline Complete or partial remission & $232(24.3)$ & $154(26.6)$ & $78(20.7)$ & \\
\hline Newly diagnosed & $382(40.0)$ & $238(41.2)$ & $144(38.2)$ & \\
\hline Other & $340(35.6)$ & $185(32.1)$ & $155(41.1)$ & \\
\hline Days since diagnosis, median [IQR] & 169 [7-965] & $163[7-1015]$ & 174 [10-942] & 97 \\
\hline Allogeneic BMT/HSCT recipient, $n$ (\%) & $146(14.5)$ & $70(11.4)$ & $76(19.3)$ & 3 \\
\hline Long course corticosteroids, n (\%) & $381(38)$ & $218(35.7)$ & $163(41.6)$ & 5 \\
\hline Charlson comorbidity index, median [IQR] & $4[3-6]$ & $4[2-5]$ & $4[3-6]$ & 1 \\
\hline Poor PS (> 2), n (\%) & $198(19.8)$ & $85(13.9)$ & $113(28.8)$ & 6 \\
\hline Reason for ICU admission, $n$ (\%) & & & & 55 \\
\hline Acute respiratory failure & $374(39.2)$ & $205(35.6)$ & $169(44.8)$ & \\
\hline Sepsis or septic shock & $255(26.8)$ & $166(28.8)$ & $89(23.6)$ & \\
\hline Metabolic disorder or acute kidney injury & $111(11.6)$ & $73(12.7)$ & $38(10.1)$ & \\
\hline Coma & $69(7.2)$ & $41(7.1)$ & $28(7.4)$ & \\
\hline Other & $144(15.5)$ & $91(15.8)$ & $53(14.1)$ & \\
\hline Organ failure, $n(\%)$ & & & & 2 \\
\hline Respiratory & $631(62.7)$ & $344(56.1)$ & $287(73.0)$ & \\
\hline Hemodynamic & $426(42.3)$ & $236(38.5)$ & $190(48.3)$ & \\
\hline Renal & $305(30.3)$ & $171(27.9)$ & $134(34.0)$ & \\
\hline Neurological & $226(22.4)$ & $114(18.6)$ & $112(28.4)$ & \\
\hline Coagulation & $194(19.3)$ & $99(16.1)$ & $95(24.2)$ & \\
\hline Hepatic & $83(8.3)$ & $39(6.4)$ & $44(11.2)$ & \\
\hline Multi-organ & $549(54.5)$ & $289(47.1)$ & $260(66.0)$ & \\
\hline SOFA score, median [IQR] & $6[3-9]$ & $5[3-7]$ & $7[5-11]$ & 1 \\
\hline
\end{tabular}

BMT bone marrow transplantation, HSCT hematopoietic stem-cell transplantation, ICU intensive care unit, IQR interquartile range, PS performance status, SOFA Sequential Related Organ Failure Assessment

Benefit from appropriate and prompt ICU management is an established fact in myocardial infarction [9], ischemic stroke [10] or major trauma [11]. Along this line, early ICU admission of patients with some physiological derangement, before organ dysfunction actually develops, may be beneficial. Chalfin et al. showed that among 50,322 patients admitted to the ICU from the ED, those admitted with $a \geq 6$ h delay had higher increased hospital length of stay (7 vs. 6 days,
$P<0.001)$ and higher ICU and hospital mortality rates (10.7\% vs. $8.4 \%, P<0.01$ and $17.4 \%$ vs. $12.9 \%$, respectively, $P<0.001)[12$. This is also in agreement with the Renaud et al. study where patients with communityacquired pneumonia had different survival according to whether they were admitted to the ICU early, late or not admitted to the ICU (adjusted OR 2.63; 95\% CI 1.42-4.90 for patients admitted early to the ICU) [13]. 
Table 2 Modalities of ICU admission and hospital mortality

\begin{tabular}{|c|c|c|c|c|}
\hline \multirow[t]{2}{*}{ Characteristics } & \multirow{2}{*}{$\begin{array}{l}\text { Overall cohort } \\
(N=1008)\end{array}$} & \multicolumn{2}{|c|}{ Status at hospital discharge } & \multirow[t]{2}{*}{ Missing data } \\
\hline & & Alive $(n=613)$ & Dead $(n=395)$ & \\
\hline $\begin{array}{l}\text { Days between hospitalization and first call to inten- } \\
\text { sivist, median [IQR] }\end{array}$ & $4[0-17]$ & $2[0-14]$ & $7[1-21]$ & 44 \\
\hline $\begin{array}{l}\text { Days between first call to intensivist and ICU admis- } \\
\text { sion, median [IQR] }\end{array}$ & $0[0-0]$ & $0[0-0]$ & $0[0-0]$ & 5 \\
\hline ICU admission requested by, $n(\%)$ & & & & 12 \\
\hline Emergency physician & $219(22.0)$ & $150(24.7)$ & $69(17.7)$ & \\
\hline Other & $777(78.0)$ & $456(75.2)$ & $321(82.3)$ & \\
\hline Experience of the physician requesting ICU, $n(\%)$ & & & & 25 \\
\hline Senior physician & $651(66.2)$ & $387(64.3)$ & $264(69.3)$ & \\
\hline Fellow & $170(17.3)$ & $112(18.6)$ & $58(15.2)$ & \\
\hline Resident/intern & $162(16.5)$ & $103(17.1)$ & $59(15.5)$ & \\
\hline Number of calls before ICU admission, $n(\%)$ & & & & 151 \\
\hline 1 & $752(87.7)$ & $474(88.9)$ & $278(85.8)$ & \\
\hline$\geq 2$ & $105(12.3)$ & $59(11.1)$ & $46(14.2)$ & \\
\hline Direct ICU admission from the ED, $n(\%)$ & $266(26.4)$ & $182(29.7)$ & $84(21.3)$ & 0 \\
\hline ICU length of stay, median [IQR], days & $5[2-11]$ & $5[2-9]$ & $5[2-13]$ & 0 \\
\hline Hospital length of stay, median [IQR], days & $28[13-47]$ & $29[16-47]$ & $25[9-47]$ & 39 \\
\hline Death at ICU discharge, $n(\%)$ & $279(27.7)$ & $0(0)$ & $279(70.6)$ & 0 \\
\hline
\end{tabular}

$E D$ emergency department, ICU intensive care unit, IQR interquartile range

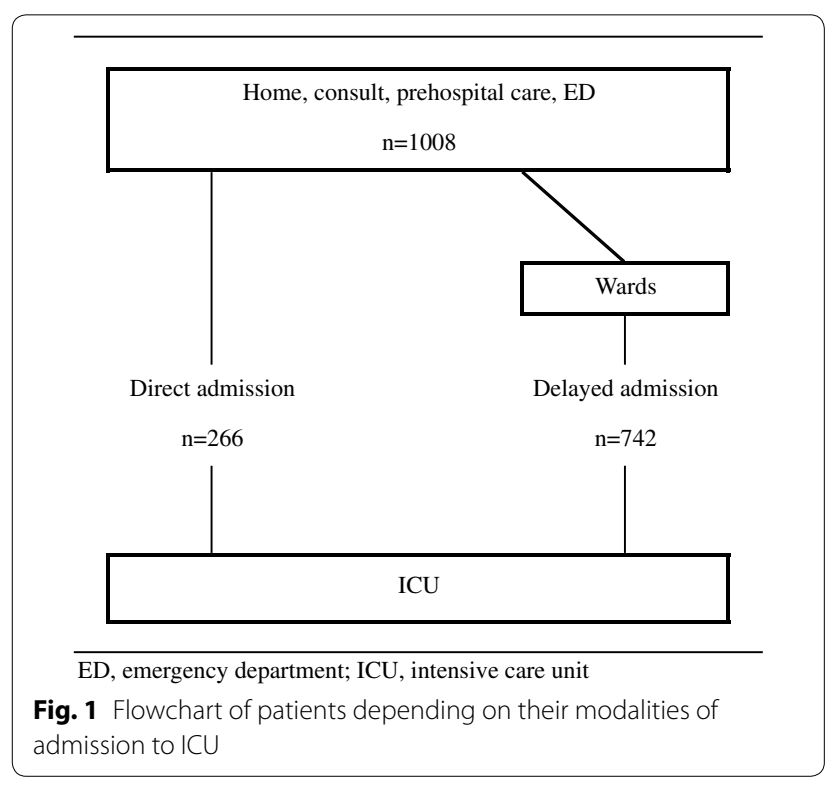

The use of ICU resources needs to be carefully discussed, however. Bed availability remains a major determinant of outcomes in settings where ICU resources are scarce [14]. Similarly, Cardoso et al. [15] found that delayed admission of patients requiring ICU management but for whom no bed was available had worse outcome than patients admitted immediately.
They found that each waiting hour was independently associated with a $1 \%$ increase in risk of hospital death (hazard ratio $=1.010 ; 95 \%$ CI 1.002 to 1.018 ).

Patients with subtle physiological derangements that could foreshadow organ failure must be close monitored so as to prevent secondary deterioration. Hence, it may be hazardous to have these patients transferred to the wards. In a cohort of 841 septic patients transferred from the ED to the wards, Whittaker et al. [16] reported that $12.5 \%$ of patients were transferred to the ICU within $48 \mathrm{~h}$ and/or died within 28 days of admission. In this study, cancer was an independent risk factor of adverse outcome. Other studies have shown that septic patients admitted to ICU from wards had higher mortality rates $[17,18]$. Last, in a study of cancer patients for whom an ICU admission was requested, $20 \%$ of the patients transferred to the wards because they were "too well" died [19]. This study suggests that ICU admission might have been inappropriately delayed and that delayed admission is associated with a high mortality rate.

Benefits from early ICU admission have also been reported in high-risk patients with malignancies and acute respiratory failure. In a prospective study on 219 patients with cancer admitted to the ICU for acute respiratory failure in France conducted by Mokart et al. [20], time between respiratory symptoms onset and ICU admission of at least 2 days was an independent predictor 
Table 3 Multivariable analysis. Variables independently associated with hospital mortality

\begin{tabular}{|c|c|c|c|c|c|c|}
\hline \multirow[t]{2}{*}{ Variables } & \multicolumn{3}{|c|}{ Model without imputation $(N=898)$} & \multicolumn{3}{|c|}{ Model with imputation $(N=1008)$} \\
\hline & OR & $95 \% \mathrm{Cl}$ & $P$ & OR & $95 \% \mathrm{Cl}$ & $P$ \\
\hline Direct admission to the ICU from the ED & 0.64 & (0.45 to 0.92$)$ & 0.02 & 0.63 & (0.45 to 0.88$)$ & 0.007 \\
\hline Age $>60$ years & 1.47 & (1.04 to 2.10$)$ & 0.03 & 1.47 & (1.05 to 2.04$)$ & 0.02 \\
\hline \multicolumn{7}{|l|}{ Disease status } \\
\hline Remission or newly diagnosed & 1.00 & & & & & \\
\hline Other & 1.49 & (1.08 to 2.06$)$ & 0.01 & 1.52 & (1.12 to 2.07$)$ & 0.008 \\
\hline Allogeneic BMT/HSCT recipient & 2.46 & (1.57 to 3.86$)$ & $<0.0001$ & 2.42 & $(1.58$ to 3.71$)$ & $<0.0001$ \\
\hline Charlson (/point) & 1.06 & (0.99 to 1.14$)$ & 0.10 & 1.07 & (1.00 to 1.15$)$ & 0.04 \\
\hline Poor PS (>2) & 1.88 & (1.30 to 2.72$)$ & $<0.001$ & 1.99 & (1.40 to 2.83$)$ & 0.0001 \\
\hline SOFA score (/point) & 1.24 & (1.19 to 1.29$)$ & $<0.00001$ & 1.23 & (1.19 to 1.28$)$ & $<0.00001$ \\
\hline \multicolumn{7}{|l|}{ Reason for ICU admission } \\
\hline Sepsis or septic shock & 1.00 & & & & & \\
\hline Acute respiratory failure & 2.16 & (1.47 to 3.2$)$ & $<0.001$ & 2.11 & (1.45 to 3.06$)$ & $<0.0001$ \\
\hline Coma & 1.68 & (0.89 to 3.15$)$ & 0.10 & 1.72 & (0.94 to 3.15$)$ & 0.08 \\
\hline Metabolic disorder or acute kidney injury & 2.05 & (1.17 to 3.56$)$ & 0.01 & 2.12 & (1.24 to 3.62$)$ & 0.006 \\
\hline Other & 2.17 & (1.30 to 3.63$)$ & 0.003 & 2.25 & (1.38 to 3.67$)$ & 0.001 \\
\hline
\end{tabular}

BMT bone marrow transplantation, ED emergency department, HSCT hematopoietic stem-cell transplantation, ICU intensive care unit, PS performance status, SOFA Sequential-Related Organ Failure Assessment

of day-28 mortality (OR: 2.65 ; $95 \%$ CI $1.29-5.44)$. In the present study, neither the delay between ICU request and ICU admission nor the number of calls before ICU admission was associated with in-hospital mortality by univariable analyses. These two variables were not included in the logistic regression model because of their colinearity with our variable of interest. Early detection of physiological derangement to allow prompt ICU admission is crucial and may be compromised in wards where surveillance is not optimal. Dedicated emergency teams have shown to be beneficial in case of soon intervention. After implementation of a medical emergency team in a comprehensive cancer center in Seoul [21], Song et al. [2] showed that in-hospital mortality rates increased significantly with increasing quartiles of time from physiological derangement to medical emergency team intervention in 199 critically ill cancer patients admitted to the ICU. Late intervention after $1.5 \mathrm{~h}$ after physiological derangement was significantly associated with in-hospital mortality in the multivariable analysis with adjustment for propensity score (OR: 3.91; 95\% CI 1.93 to 7.94$)$. These results remained true for long-term mortality in a study including 525 patients with cancer where early intervention was significantly associated with 1 -year mortality after adjusting for potential confounders (hazard ratio: 0.456 ; $95 \%$ CI 0.348 to 0.597 ) [22].

Deciding the accurate timing for ICU admission may be challenging. ICU referral or admission decisions may be even more difficult in cancer patient when prognosis is uncertain [23]. Studies to incite early ICU management in high-risk patients are warranted. Besides survival benefits, organ dysfunction recovery and ability to receive optimal cancer care are critical endpoints of such interventions.

\section{Limitations}

Our study has several limitations. First, this was a post hoc analysis of a cohort study dealing with the comparison of non-randomized groups. Obviously, because randomizing modalities of ICU admission may be complex, baseline characteristics of patients admitted from wards differ from those admitted directly from the ED. To handle these differences and limit selection bias, we performed a multivariable logistic regression to incorporate differences in baseline characteristics of these groups; however, it is possible that non-observed confounding factors exist such as hospital bed availability. Secondly, estimated benefit disappeared in the propensity score-matched sample, but this may be at least partially due to a lack of power given the limited sample size (402 vs. 1008) and on differences between matched and unmatched patients resulting in a selected population. However, variables that differed between both groups were already part of the multivariable logistic regression, except the type of underlying malignancy and the use of long-term corticosteroids. Thus, we reran a second multivariable analysis including these two variables and direct ICU admission remained associated with a better outcome. Third, we focus our research on patients with hematological malignancies admitted to the ICU and did 
Unadjusted $(\mathrm{N}=1008) \quad 0.64[0.47-0.86]$

Adjusted, after multiple imputation $(\mathrm{N}=1008) 0.63[0.45-0.88]$

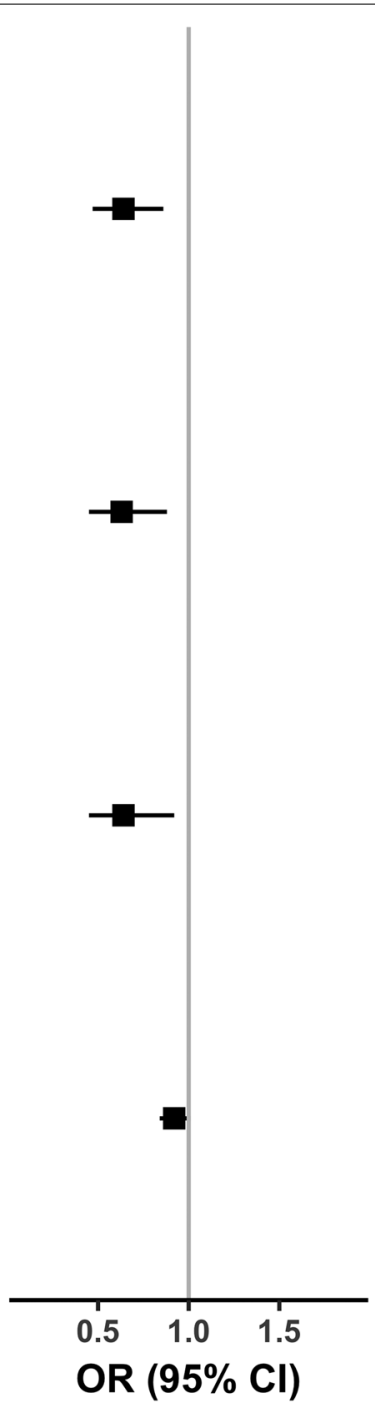

Fig. 2 Effects of direct ICU admission from the ED on hospital mortality

not consider patients admitted to the wards who did not require or who were not referred to the ICU. Furthermore, patients admitted to the ICU from wards were not necessarily managed in the ED before. Fourth, we could not assess the time elapsed between the need for and the actual ICU admission. It could have been interesting to determine if patients admitted in wards had longer delays between first symptoms and ICU admission reflecting flaws in monitoring. Fifth, we did not consider the outcomes after hospital discharge. It is possible that a substantial part of these patients was transferred to palliative care unit lowering the benefit of survival at discharge.

\section{Conclusions}

In summary, in this post hoc study, patients with hematological malignancies admitted to the ICU were more likely to be alive at hospital discharge if they were directly admitted from the ED rather than from the wards. We are not yet to pretend that patients admitted to the ICU from wards who were previously managed in the ED should have been directly admitted from there. We, however, put forward that these high-risk patients need prompt specific management and close monitoring when they develop subtle physiological derangement to anticipate organ dysfunctions. This close monitoring may be inadequate in the wards and should be achieved by specialized teams in intensive care or high dependency units. Assessment of alarm signs and early predictors of poor outcome 


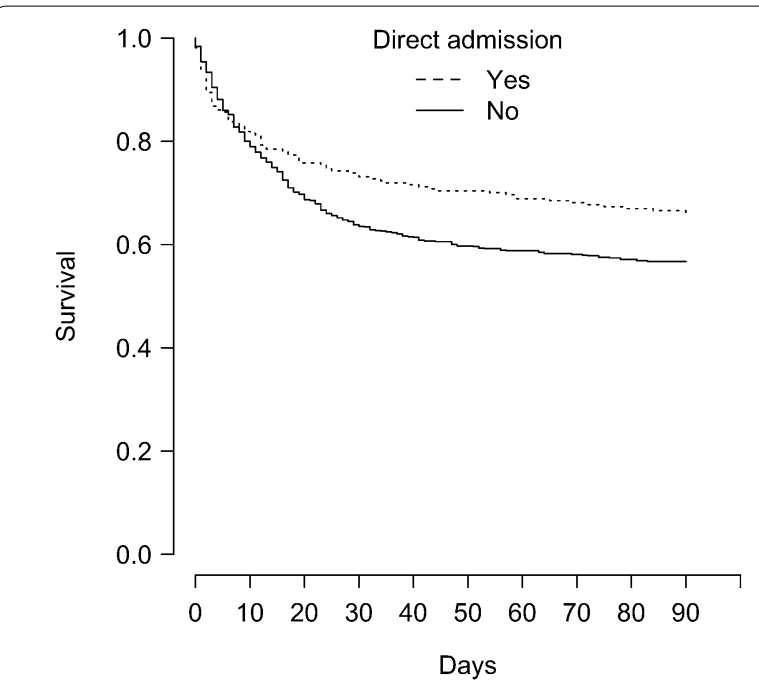

Fig. 3 Kaplan-Meier survival during 90 days from intensive care unit admission depending on direct admission from the emergency department

in cancer patients admitted to the ED is crucial so as to allow early referral to the ICU and avoid delays in treatment initiation and mis-orientation.

\section{Supplementary information}

Supplementary information accompanies this paper at https://doi. org/10.1186/s13613-019-0587-7.

Additional file 1. Univariable analysis. Variables associated with hospita mortality. BMT bone marrow transplantation, CLL chronic lymphocytic leukemia, CML chronic myeloid leukemia, ED emergency department, HSCT hematopoietic stem-cell transplantation, ICU intensive care unit, MDS myelodysplastic syndrome, PS performance status, SOFA Sequential Related Organ Failure Assessment.

Additional file 2. Comparison of patient characteristics according to direct ICU admission. ALL acute lymphocytic leukemia, AML acute myeloid leukemia, BMT bone marrow transplantation, CLL chronic lymphocytic leukemia, CML chronic myeloid leukemia, ED emergency department, HSCT hematopoietic stem-cell transplantation, ICU intensive care unit, IQR interquartile range, MDS myelodysplastic syndrome, $P S$ performance status, SOFA Sequential Related Organ Failure Assessment.

Additional file 3. Loveplot. Absolute mean differences in patient charac teristics before (unadjusted) and after (adjusted) matching on propensity score. BMT bone marrow transplantation, CLL chronic lymphocytic leukemia, CML chronic myeloid leukemia, HSCT hematopoietic stem-cell transplantation, ICU intensive care unit, MDS myelodysplastic syndrome, PS performance status, SOFA Sequential Related Organ Failure Assessment.

\section{Abbreviations}

ALL: acute lymphocytic leukemia; AML: acute myeloid leukemia; BMT: bone marrow transplantation; CLL: chronic lymphocytic leukemia; CML: chronic myeloid leukemia; ED: emergency department; HSCT: hematopoietic stemcell transplantation; ICU: intensive care unit; IQR: interquartile range; MDS: myelodysplastic syndrome; PS: performance status; SOFA: Sequential-related Organ Failure Assessment.

\section{Acknowledgements}

Not applicable.

\section{Authors' contributions}

OP, SC and EA conceived the study, analyzed the data and wrote the manuscript. APM, PP, AK, FP, DM, VL, AD, MN, FB, CL, DB, AM and EA were involved in provision of study materials or patients, collection and assembly of data. All authors read and approved the final manuscript.

\section{Funding}

The study was supported by Grant PHRC AOM 08235 from the French Ministry of Health.

\section{Availability of data and materials}

The datasets generated and analyzed during the current study are not publicly available due to the potential risk of leakage of personally identifiable information

\section{Ethics approval and consent to participate}

The study was approved by the appropriate ethics committees in France and Belgium.

\section{Consent for publication}

Not applicable.

\section{Competing interests}

The authors declare that they have no competing interests.

\section{Author details}

${ }^{1}$ Emergency Department, Hôpital Saint-Louis, 1 avenue Claude Vellefaux, 75010 Paris, France. ${ }^{2}$ Biostatistics and Medical Information Department, Hôpital Saint-Louis, Paris, France. ${ }^{3}$ Centre de Recherche en Épidémiologie et Statistiques - Université de Paris (CRESS-INSERM-UMR1153), Epidemiology and Clinical Statistics for Tumor, Respiratory, and Resuscitation Assessments (ECSTRRA) Team, Paris, France. ${ }^{4}$ Université de Paris, Paris, France. ${ }^{5}$ Intensive Care Unit, Institut Jules Bordet, Université libre de Bruxelles (ULB), Brussels, Belgium. ${ }^{6}$ Intensive Care Unit, Hôpital Brabois, Vandoeuvre Les Nancy, France. ${ }^{7}$ Intensive Care Unit, Centre hospitalier régional universitaire, Angers, France. ${ }^{8}$ Intensive Care Unit, Hôpital Cochin, Paris, France. ${ }^{9}$ Institut Cochin, INSERM U1016, CNRS UMR 8104, Paris, France. ${ }^{10}$ Intensive Care Unit, Institut Paoli Calmettes, Marseille, France. ${ }^{11}$ Intensive Care Unit, Hôpital Saint-Louis, Paris, France. ${ }^{12}$ Intensive Care Unit, Hôpital Pitié-Salpêtrière, Paris, France. ${ }^{13}$ INSERM, UMRS 1158 Neurophysiologie respiratoire expérimentale et clinique, Paris, France. ${ }^{14}$ Université Paris Sorbonne, Paris, France. ${ }^{15}$ Intensive Care Unit, Hôpital Victor Provo, Roubaix, France. ${ }^{16}$ Intensive Care Unit, Hôpital André Mignot, Versailles, France. ${ }^{17}$ Intensive Care Unit, Centre hospitalier départemental Vendee, La Roche Sur Yon, France. ${ }^{18}$ Intensive Care Unit, Hôpital universitaire de Ghent, Ghent, Belgium.

Received: 25 April 2019 Accepted: 21 September 2019

Published online: 02 October 2019

\section{References}

1. Doukhan L, Bisbal M, Chow-Chine L, et al. Respiratory events in ward are associated with later intensive care unit (ICU) admission and hospital mortality in onco-hematology patients not admitted to ICU after a first request. PLOS ONE. 2017;12:e0181808.

2. Song JU, Suh GY, Park HY, et al. Early intervention on the outcomes in critically ill cancer patients admitted to intensive care units. Intensive Care Med. 2012:38:1505-13.

3. Azoulay E, Mokart D, Pène F, et al. Outcomes of critically ill patients with hematologic malignancies: prospective multicenter data from France and Belgium - a groupe de recherche respiratoire en réanimation oncohématologique study. J Clin Oncol. 2013;31:2810-8.

4. Chevret S, Seaman S, Resche-Rigon M. Multiple imputation: a mature approach to dealing with missing data. Intensive Care Med. 2015;41:348-50.

5. White $I R$, Royston P, Wood AM. Multiple imputation using chained equations: issues and guidance for practice. Stat Med. 2011;30:377-99. 
6. Franklin JM, Rassen JA, Ackermann D, et al. Metrics for covariate balance in cohort studies of causal effects. Stat Med. 2014;33:1685-99.

7. Austin PC. A comparison of 12 algorithms for matching on the propensity score. Stat Med. 2014;33:1057-69.

8. Therneau TM, Grambsch PM. Testing proportional hazards. In: Therneau TM, Grambsch PM, editors. Modeling survival data: extending the cox model. New York: Springer; 2000. p. 127-52.

9. Ibanez B, James S, Agewall S, et al. 2017 ESC guidelines for the management of acute myocardial infarction in patients presenting with ST-segment elevation: the Task Force for the management of acute myocardial infarction in patients presenting with ST-segment elevation of the European Society of Cardiology (ESC). Eur Heart J. 2018;39:119-77.

10. Lees KR, Bluhmki E, von Kummer R, et al. Time to treatment with intravenous alteplase and outcome in stroke: an updated pooled analysis of ECASS, ATLANTIS, NINDS, and EPITHET trials. Lancet. 2010;375:1695-703.

11. Spahn DR, Bouillon B, Cerny V, et al. Management of bleeding and coagulopathy following major trauma: an updated European guideline. Crit Care. 2013;17:R76.

12. Chalfin DB, Trzeciak S, Likourezos A, et al. Impact of delayed transfer of critically ill patients from the emergency department to the intensive care unit. Crit Care Med. 2007;35:1477-83.

13. Renaud B, Brun-Buisson C, Santin A, et al. Outcomes of early, late, and no admission to the intensive care unit for patients hospitalized with community-acquired pneumonia. Acad Emerg Med. 2012;19:294-303.

14. Robert R, Reignier J, Tournoux-Facon C, et al. Refusal of intensive care unit admission due to a full unit: impact on mortality. Am J Respir Crit Care Med. 2012:185:1081-7.

15. Cardoso LT, Grion CM, Matsuo T, et al. Impact of delayed admission to intensive care units on mortality of critically ill patients: a cohort study. Crit Care. 2011;15:R28
16. Whittaker SA, Fuchs BD, Gaieski DF, et al. Epidemiology and outcomes in patients with severe sepsis admitted to the hospital wards. J Crit Care. 2015:30:78-84.

17. Vincent JL, Rello J, Marshall J, et al. International study of the prevalence and outcomes of infection in intensive care units. JAMA. 2009;302:2323-9.

18. Brun-Buisson C, Meshaka P, Pinton P, et al. EPISEPSIS: a reappraisal of the epidemiology and outcome of severe sepsis in French intensive care units. Intensive Care Med. 2004;30:580-8.

19. Thiéry G, Azoulay E, Darmon M, et al. Outcome of cancer patients considered for intensive care unit admission: a hospital-wide prospective study. J Clin Oncol. 2005;23:4406-13.

20. Mokart D, Lambert J, Schnell D, et al. Delayed intensive care unit admission is associated with increased mortality in patients with cancer with acute respiratory failure. Leuk Lymphoma. 2013;54:1724-9.

21. Lim SY, Park SY, Park HK, et al. Early impact of medical emergency team implementation in a country with limited medical resources : a beforeand-after study. J Crit Care. 2011;26:373-8.

22. Lee DS, Suh GY, Ryu JA, et al. Effect of early intervention on long-term outcomes of critically ill cancer patients admitted to ICUs. Crit Care Med. 2015:43:1439-48.

23. Azoulay $E$, Pène F, Darmon $M$, et al. Managing critically ill hematology patients: time to think differently. Blood Rev. 2015;29:359-67.

\section{Publisher's Note}

Springer Nature remains neutral with regard to jurisdictional claims in published maps and institutional affiliations.

\section{Submit your manuscript to a SpringerOpen ${ }^{\circ}$ journal and benefit from:}

- Convenient online submission

- Rigorous peer review

- Open access: articles freely available online

- High visibility within the field

- Retaining the copyright to your article

Submit your next manuscript at springeropen.com 